\title{
Investments in next generation access infrastructures under regulatory uncertainty
}

\author{
Markos Tselekounis, Dimitris Varoutas* \\ Department of Informatics and Telecommunications, University of Athens, Panepistimiopolis, Ilissia, Athens 15784, Greece
}

\section{A R T I C L E I N F O}

Available online 19 July 2013

\section{Keywords:}

Access regulation

Dynamic efficiency

Investment incentives

Regulatory uncertainty

Social welfare

Telecommunications

\begin{abstract}
A B S T R A C T
This article studies the impact of regulatory uncertainty on an incumbent's incentives to undertake the socially optimal investments in NGA networks. Thus, a regulatory noncommitment setting in which the regulator sets the access price after the deployment of the NGA network is used. In particular, it is assumed that the regulator sets the access price at the marginal cost of providing the access with some probability and gives an access markup, which equals the average cost of the investments, with the complementary probability. It is found that when the slope of the marginal investment cost function is not particularly steep in relation to the impact of investments on demand, the incumbent underinvests compared to the socially optimal investment level. On the contrary, in a more realistic case when the impact of investments on demand is low in relation to the slope of the marginal investment cost function, the incumbent may overinvest or underinvest depending on the probability of incorporating an access markup into the access price.
\end{abstract}

(c) 2013 Elsevier Ltd. All rights reserved.

\section{Introduction}

The migration from the legacy copper access networks to fiber access networks capable of providing high-speed broadband services (hereafter referred to as Next Generation Access (NGA) networks ${ }^{1}$ ) has induced a growing interest in the relationship between access regulation and investment incentives. ${ }^{2}$ The related literature concludes that the regulators' two-fold goal to foster competition and encourage efficient and timely investments in NGA networks is related to the common trade-off between static and dynamic efficiency.

In particular, mandated access at cost-based prices reduces the use of monopoly power over the access infrastructure by preventing the incumbent from foreclosing the entrants from the downstream (retail) market. Cost-based access regulation thus leads to sustainable service-based competition within one network, and hence, improves static efficiency (Bouckaert, van Dijk, \& Verboven, 2010; Valletti, 2003). However, mandating the access at cost-based prices discourages both incumbents and potential entrants to invest in new access infrastructures (Jorde, Sidak, \& Teece, 2000). According to Cave and Prosperetti (2001), the reason for this negative relationship between access regulation and incumbents' incentives to invest is that the incumbents anticipate that they will be required to offer access to their rivals at cost-based prices. Therefore, potential entrants, who can free-ride on the incumbent's network, will wait for the incumbent to invest in new

\footnotetext{
* Corresponding author. Tel.:+30 2107275318 , Fax.:+30 2107275214.

E-mail addresses: markos@di.uoa.gr (M. Tselekounis),D.Varoutas@di.uoa.gr, dvaroutas@gmail.com (D. Varoutas).

${ }^{1}$ According to EU Commission (2010) NGA networks means wired access networks which consist wholly or in part of optical elements and which are capable of delivering broadband access services with enhanced characteristics (such as higher throughput) as compared to those provided over already existing copper networks. In most cases NGAs are the result of an upgrade of an already existing copper or coaxial access network.

${ }^{2}$ See Cambini and Jiang (2009) for a recent and comprehensive review of the theoretical and empirical literature on the relationship between broadband investment and regulation.
} 
access infrastructures and then seek access (Valletti, 2003). The conclusion is that cost-based access regulation, which is limited to promote service-based competition, leads to losses in dynamic efficiency (Bouckaert et al., 2010).

For this reason, the ongoing research on this area is gradually shifting its focus from assessing the efficiency implications of cost-oriented access schemes to proposing new regulatory approaches that may promote both static and dynamic efficiency. This implies that access regulation of NGA services is still considered necessary for inducing socially optimal investments. In this context, a first set of papers studies the impact of an investment-contingent access price on investment incentives and competition. Henriques (2011) and Sauer (2011) show that contrary to a fixed access charge, an access fee that is contingent on firms' (non-overlapping) investments can implement the socially efficient investment level. This outcome holds either if the access charge depends on the investments of both the incumbent and the entrant (former article) or on each operator's own investment level (latter article).

Nitsche and Wiethaus (2011) introduce uncertainty about the success of NGA investments and focus on an incumbent's investment incentives in order to study the efficiency outcomes of different regulatory regimes. They show that under an access price that spreads investment costs over total output quantities (i.e. an investment-contingent access scheme), a regime with fully distributed costs or a regulatory holiday induce highest investments, followed by risk-sharing and forward-looking cost-based regulation. In addition, combining strong competitive intensity with reasonable investment incentives indicates that a risk sharing approach induces highest consumer surplus, followed by regimes with fully distributed costs, regulatory holiday and forward-looking cost-based regulation. Bender (2011) extends the work of Nitsche and Wiethaus by introducing horizontal product differentiation and assuming that the entrant may not share the investment costs with the incumbent but bears a fixed part of the investment costs (i.e. a cooperation regime). The author finds that there is no single regime which always yields highest investments as full distribution, as well as, cooperation might maximize the investment incentives, whereas the optimal regulatory policy is mainly subject to the degree of product differentiation.

It is important to note that the abovementioned articles, which are based on an investment-contingent access price, assume that the regulator can make ex ante credible commitments. This implies that the regulator sets the access price prior to the investments, and hence, the firms invest under regulatory certainty. Although this modeling setup is consistent with the European Commission (EC) Recommendation on regulated access to NGA (EU Commission, 2010), it is socially not optimal for the regulator to make ex ante commitments for an unreasonably long regulatory period (WIK, 2009). Therefore, in providing greater regulatory certainty the regulator has to make another trade-off between the positive effects of greater certainty on investment incentives and possible negative effects of erroneous intervention on welfare (OPTA, 2010).

As a result, a second set of papers studies the impact of access regulation on investment incentives under regulatory noncommitment. In this case, it is assumed that the regulator cannot make ex ante credible commitments, and hence, the firms invest prior to the regulation of the access. Foros (2004) studies the impact of cost-based access regulation on an incumbent's incentives to invest in network upgrade in the presence of spillover effects. It is found that when the incumbent is much more efficient in providing value-added services than the entrant, then the incumbent can foreclose the entrant from the market by overinvesting in quality. In addition, Kotakorpi (2006) points out that, under cost-based regulation, the incumbent underinvests in relation to the socially optimal level. Mizuno and Yoshino (2012) allow an enlarged range of spillovers in order to examine the impact of regulatory non-commitment on the properties of a welfare maximizing access charge. They find that when the degree of spillover is small and the incumbent's investment cost is high (respectively, low), the incumbent has an incentive to utilize regulatory non-commitment to induce a high (respectively, low) access charge by overinvesting (respectively, underinvesting) in infrastructure.

Contrary to the three previous articles that use a fixed access charge, Klumpp and Su (2010) assume an investmentcontingent access price which is revenue-neutral. This implies that each downstream firm contributes to the depreciation of the investment costs according to its market share. They show that, under this rule, the incumbent chooses a higher investment level compared to that of a monopolist and its investment incentives increases with the number of downstream competitors. Thus, they argue that a policy of revenue-neutral open access can increase both static and dynamic efficiency. Sarmento and Brandao (2007) compare the investment and competition outcomes of an access price which equals the marginal cost of providing the access plus the average cost of the investments with those derived by the retail-minus regulation and the deregulation of the access price. They conclude that retail-minus regulation leads to better results than cost-based regulation in terms of investment level and consumer surplus as long as the regulator carefully defines the retailminus instrument.

Last, Cambini and Silvestri (2012) also study the impact of regulatory non-commitment on the incumbent's investment incentives, but their research focuses on the timing of the investments rather than on the extent of NGA deployment. For this reason, they use a dynamic setting with demand uncertainty in order to compare the efficiency outcomes of three different regulatory regimes: full regulation (the NGA network is regulated), partial regulation (the NGA network is unregulated) and risk sharing (fixed investment costs are shared but there are no side payments between firms for the use of the NGA). They conclude that the investment is always undertaken later than in the social optimum in all regulatory regimes.

It can be thus concluded that the related literature provides useful results concerning the effectiveness of particular access pricing schemes and certain regulatory regimes on promoting both static and dynamic efficiency. However, the articles that examine the relationship between access regulation and investment incentives under regulatory noncommitment take the regulator's decision as given. As a result, they fail to take into account the fact that there is 
uncertainty about the access pricing formula once the investments are in place. In particular, some articles (Foros, 2004; Kotakorpi, 2006; Mizuno \& Yoshino, 2012) assume that the firms anticipate that the regulator will set the welfare maximizing access price, whereas others (Klumpp \& Su, 2010; Sarmento \& Brandao, 2007) assume that the investmentcontingent access price is ex ante known. Therefore, the difference in the results of the commitment and the noncommitment models is mainly driven by the sequential timing of the games rather than by the regulatory uncertainty of the non-commitment games.

On the contrary, this paper takes into account the regulator's incentives to deviate from an investment-contingent access pricing rule (which implies that the regulator compensates the incumbent for the investment risks) by setting the social welfare maximizing access price once the investments are in place. In particular, it is assumed that the regulator may implement the access rule proposed by Sarmento and Brandao (2007) or set the welfare maximizing access price. This implies that when the incumbent invests prior to the regulation of the access, there is an uncertainty about the level of the access charge. The aim of this paper is to model such regulatory uncertainty and to study its impact on an incumbent's investment incentives and on the subsequent welfare outcomes.

It is found that when the probability of compensating the incumbent for the investment risks increases, the incumbent's investment incentives increase and the socially optimal investment level decreases. In addition, the critical value of such probability that makes the incumbent undertake the socially optimal (efficient) investments is decreasing in the slope of the marginal investment cost function and increasing in the effect of the investments on demand. Thus, when the slope of the marginal investment cost function is not particularly steep in relation to the positive impact of investments on demand, the incumbent always underinvests compared to the socially optimal investments. On the contrary, in a more realistic case, when the impact of investments on demand is low in relation to the slope of the marginal investment cost function, the incumbent overinvests for high probability of setting the access pricing rule of Sarmento and Brandao (2007) and underinvests for low probability values.

It is thus obvious that the effectiveness of access price regulation on promoting both static and dynamic efficiency (i.e. inducing the socially optimal investments in NGA networks) does not only depend on the underlying demand and cost structure (as the related literature concludes), but is also dependent on the expected probability of a regulatory deviation from a particular investment-contingent access pricing rule.

The rest of the paper is organized as follows. Section 2 gives an outline of the basic assumptions and definitions of the model. Section 3 provides the equilibrium of the game when the regulatory uncertainty is taken into account. Section 4 compares the privately and the socially optimal investment levels derived in Section 3 in order to draw regulatory implications. The last section summarizes the key findings of this article.

\section{The model}

This section presents a simple model used in order to assess the impact of regulatory uncertainty on an incumbent's incentives to undertake the socially optimal investments in new access infrastructures. In particular, the model used in this paper is quite similar to that of Sarmento and Brandao (2007) in terms of demand and cost structure, as well as, the timing of the game.

\subsection{Demand side}

The retail (downstream) market is characterized as an unregulated duopoly market in which the incumbent (the subsidiary firm of the upstream monopolist) and the entrant (the independent firm) choose quantities simultaneously and independently (i.e. firms compete á la Cournot). It is further assumed that since the prospective investors in NGA networks are for large part the former incumbent operators (OPTA, 2010), the incumbent decides its optimal NGA investment level first and then the entrant seeks access to the incumbent's NGA network. ${ }^{3}$

The inverse demand function is given by $p=1+\beta I-\left(q_{1}+q_{2}\right)$, where $p$ is the retail market price, $q_{1}$ and $q_{2}$ are the quantities supplied by the incumbent and the entrant respectively, $I$ represents the level of the NGA investments undertaken by the incumbent, and $\beta$ represents the impact of a marginal change in the investment level on the retail price (ceteris paribus). It is also assumed that $\beta>0$, which implies that an increase in the NGA investment level leads to an outward parallel shift in the demand that benefits both retailers.

\subsection{Supply side}

The NGA deployment is continuous where a larger $I$ reflects a fiber deployment closer to the consumers' premises. The incumbent faces a quadratic NGA investment cost function with respect to $I$, given by $C(I)=\varphi I^{2} / 2$, with $\varphi>0$. The cost

\footnotetext{
${ }^{3}$ This assumption reflects the EU Commission's argument that in most cases NGAs are the result of an upgrade of an already existing copper access network. The reason is that the former incumbent operators usually upgrade their access networks by replacing part of the copper networks with optical elements (i.e. FTTC variants). On the contrary, a narrower definition of NGA networks (i.e. FTTH/B) may reflect the fact that alternative operators seem to dominate such network deployment. However, the results of this paper do not change qualitatively if we assume that the entrant decides its optimal NGA investment level first and then the incumbent seeks access to the entrant's NGA network.
} 
parameter $\varphi$ represents the slope of the marginal investment cost function, and hence, as $\varphi$ increases the total investment costs increase as well (for a given investment level). For this reason, the term "investment cost parameter" refers to $\varphi$, whereas the term "investment costs" refers to the total costs of NGA investments denoted by $C(I)$. The convex form of the NGA investment cost function reflects the fact that fiber deployment becomes marginally more expensive as it is being laid down towards consumers' premises. It is further assumed that the NGA investment level does not have any impact on the (fixed) marginal cost of providing the access denoted by $c,(c<1)$. In addition, the production of one unit of the retail service requires one unit of the upstream input (fixed coefficients technology).

The access price that the entrant should pay to the incumbent in order to have access to the incumbent's NGA network is denoted by $w$, assuming $w \geq c$. The regulator defines the access price as the marginal cost of providing the access (c) plus a fraction $k$ of the total investment costs, that is $w=c+k C(I)$, with $k<1$. If $k=0$, the regulator sets the access price at the marginal cost of providing the access, whereas if $k \in(0,1)$, the regulator (partially or fully) compensates the investor for the uncertainty of NGA investments.

Last, the quality of the input sold by the monopolist is the same whether it is sold to the incumbent or to the entrant. In addition, the cost of all other inputs is equal for both retailers and normalized to zero.

\subsection{Timing of the game}

This paper falls into the literature which assumes that the regulator cannot make ex ante credible commitments on its future interventions, and hence, the incumbents decide their optimal investment level prior to the regulation of the access. Therefore, the timing of the game is as follows:

- Firstly, the incumbent decides the investment level $I$ that maximizes its profits.

- Secondly, the regulator chooses an access price $w$ that may incorporate a risk premium reflecting the uncertainty of NGA investments, i.e. $k \in[0,1)$.

- Finally, the retail price and outputs of the firms are defined by Cournot competition between downstream firms.

According to Sarmento and Brandao (2007), the regulator sets the access price equal to the marginal cost of providing the access plus the average cost of the investments. Therefore, $k=(1 / I)$ and $w=c+(\varphi I / 2)$. This definition of the access price implies that the average cost of the investments can be seen as an access markup that fully compensates the investor for the uncertainty of NGA investments. However, Cave and Prosperetti (2001), as well as, Foros (2004) argue that the regulator is expected to set the access price at the marginal cost of providing the access (i.e. $k=0$ ) in order to maximize social welfare as soon as the incumbent invests in NGA network. ${ }^{4}$ In this paper, it is assumed that the incumbent anticipates that $k=(1 / I)$ with probability $\alpha$ and $k=0$ with probability $(1-\alpha)$, where $\alpha \in[0,1] .^{5}$ In the former case, the regulator fully compensates ("FC") the incumbent for the investment risks; and in the latter case, the regulator sets the social welfare maximizing access price, which implies that the regulator does not compensate ("NC") the incumbent for the uncertainty of NGA investments.

The profit functions of the incumbent (firm 1) and the entrant (firm 2) are given, respectively, by:

$$
\begin{aligned}
& \pi_{1}=(p-c) q_{1}+(w-c) q_{2}-\varphi I^{2} / 2 \\
& \pi_{2}=(p-w) q_{2}
\end{aligned}
$$

Therefore, when $w=c+(\varphi I / 2)$, the two firms make the following profits (gross of investment costs):

$$
\begin{aligned}
& \pi_{1}^{F C}=\left[\left(P^{F C}-c\right) q_{1}^{F C}+(\varphi I / 2) q_{2}^{F C}\right] \alpha \\
& \pi_{2}^{F C}=\left[\left(P^{F C}-c-\varphi I / 2\right) q_{2}^{F C}\right] \alpha
\end{aligned}
$$

whereas, when $w=c$ the respective profit functions are (gross of investment costs):

$$
\begin{aligned}
& \pi_{1}^{N C}=\left[\left(p^{N C}-c\right) q_{1}^{N C}\right](1-\alpha) \\
& \pi_{2}^{N C}=\left[\left(p^{N C}-c\right) q_{2}^{N C}\right](1-\alpha)
\end{aligned}
$$

It is obvious that total profits of the incumbent and the entrant are given, respectively, by:

$$
\begin{aligned}
& \pi_{1}=\pi_{1}^{F C}+\pi_{1}^{N C}-\varphi I^{2} / 2 \\
& \pi_{2}=\pi_{2}^{F C}+\pi_{2}^{N C}
\end{aligned}
$$

\footnotetext{
${ }^{4}$ Indeed, it is shown in Appendix A1 that social welfare is maximized for $k<0$. However since it is generally assumed that $w \geq c$, the access price that maximizes social welfare is $w=c$.

${ }^{5}$ This is the typical way that the related literature handles the imperfect commitment to access price. For a particular example, see Vareda (2010).
} 


\section{Equilibrium of the game}

The aim of this paper is to assess the impact of regulatory uncertainty on the incumbent's incentives to invest in NGA networks and to draw regulatory implications from the comparison between the NGA investment level chosen by the incumbent (i.e. the privately optimal level) and the respective level of NGA investments that maximizes social welfare (i.e. the socially optimal level).

The backward induction technique is used to find the equilibrium of the whole game. Therefore, the analysis begins with the computation of the retail price and the output of the firms as a function of the investment level and the access price. Then, the regulator chooses an access price $w$ that may incorporate a risk premium reflecting the uncertainty of NGA investments. In particular, the access price is $w=c+(\varphi I / 2)$ with probability $\alpha$ or $w=c$ with probability $(1-\alpha)$. Hence, the profits functions of both retailers are derived as a function of the investment level and the probability $\alpha$. Taking into account the derived results, the privately and socially optimal investment levels are obtained as a function of $\alpha$.

\subsection{Retail competition}

Substituting $p=1+\beta I-\left(q_{1}+q_{2}\right)$ in Eqs. (1) and (2), and taking the first order condition with respect to $q_{1}$ and $q_{2}$, respectively, gives the reaction function of each firm to the quantity supplied by the other. Solving simultaneously the reaction functions for both operators yields the output of the firms and the subsequent retail price:

$$
\begin{aligned}
& q_{1}=(1+\beta I+w-2 c) / 3 \\
& q_{2}=(1+\beta I-2 w+c) / 3 \\
& p=(1+\beta I+w+c) / 3
\end{aligned}
$$

\subsection{Access price regulation}

In this stage the regulator sets the price that the entrant should pay to the incumbent in order to have access to the NGA network. In particular, the regulator may compensate the incumbent for the NGA investment risks or set the access price equal to the marginal cost of providing the access in order to maximize social welfare. Substituting $w=c+(\varphi I / 2)$ in Eqs. (9)-(11) gives the output of the firms and the subsequent retail price when the access price includes a markup equal to the average cost of the investments. Hence:

$$
\begin{aligned}
& q_{1}^{F C}=(1+\beta I-c+\varphi I / 2) / 3 \\
& q_{2}^{F C}=(1+\beta I-c-\varphi I) / 3 \\
& p^{F C}=(1+\beta I+2 c+\varphi I / 2) / 3
\end{aligned}
$$

On the contrary, when the regulator does not compensate the incumbent for the investment risks, the output of the firms and the subsequent retail price are derived by substituting $w=c$ in Eqs. (9)-(11). Hence:

$$
\begin{aligned}
& q_{1}^{N C}=(1+\beta I-C) / 3 \\
& q_{2}^{N C}=(1+\beta I-C) / 3 \\
& p^{N C}=(1+\beta I+2 c) / 3
\end{aligned}
$$

Given that the total output in the compensation case is given by $Q^{F C}=\left(q_{1}^{F C}+q_{2}^{F C}\right)$ and in the non-compensation case by $Q^{N C}=\left(q_{1}^{N C}+q_{2}^{N C}\right)$, the levels of total output $(Q)$, consumer surplus $(C S)$, each provider's profits, and social welfare $(S W)$ are as follows:

$$
\begin{aligned}
& Q=\alpha Q^{F C}+(1-\alpha) Q^{N C}=(2+2 \beta I-2 c-\alpha \varphi I / 2) / 3 \\
& C S=\left[\alpha\left(Q^{F C}\right)^{2}+(1-\alpha)\left(Q^{N C}\right)^{2}\right] / 2 \Rightarrow \\
& C S=\left[(1-c)(16-16 c+32 \beta I-8 \alpha \varphi I)+I^{2}\left(16 \beta^{2}-8 \alpha \beta \varphi+\alpha \varphi^{2}\right)\right] / 72 \\
& \pi_{1}=\left[(1-c)(4-4 c+8 \beta I+10 \alpha \varphi I)+2 \varphi I^{2}(5 \alpha \beta-9)+I^{2}\left(4 \beta^{2}-5 \alpha \varphi^{2}\right)\right] / 36 \\
& \pi_{2}=\left[(1-c)(1-c+2 \beta I-2 \alpha \varphi I)+I^{2}\left(\alpha \varphi^{2}-2 \alpha \beta \varphi+\beta^{2}\right] / 9\right. \\
& S W=\pi_{1}+\pi_{2}+C S \Rightarrow \\
& S W=\left[(1-c)(32-32 c+64 \beta I-4 \alpha \varphi I)+I^{2}\left(32 \beta^{2}-4 \alpha \beta \varphi-36 \varphi-\alpha \varphi^{2}\right)\right] / 72
\end{aligned}
$$




\subsection{Privately optimal investments}

Taking the first order condition of Eq. (20) with respect to $I$, gives the NGA investment level that maximizes the incumbent's (private) profits:

$$
I^{*}=(1-c)(4 \beta+5 \alpha \varphi) /\left(18 \varphi+5 \alpha \varphi^{2}-4 \beta^{2}-10 \alpha \beta \varphi\right)
$$

It is obvious that, as long as $X \equiv 18 \varphi+5 \alpha \varphi^{2}-4 \beta^{2}-10 \alpha \beta \varphi>0$, the incumbent's profit is a concave function of $I$, and hence, there exists a unique equilibrium in which the incumbent chooses a positive NGA investment level. In addition, taking the first derivative of Eq. (23) with respect to $\alpha$ yields:

$$
\partial I^{*} / \partial \alpha=10 \varphi(1-c)\left(9 \varphi+2 \beta^{2}-2 \beta \varphi\right) /\left(X^{2}\right)>0
$$

From Eq. (24) it can be deduced that as $\alpha$ increases, the incumbent chooses a higher NGA investment level. In other words, a higher certainty about the compensation of the incumbent leads to a higher NGA deployment. In addition, the level of social welfare that corresponds to the privately optimal investments is derived by substituting $I^{*}$ into Eq. (22). Therefore:

$$
\begin{aligned}
S W^{*}= & \varphi(1-c)^{2}\left(100 \alpha^{3} \beta \varphi^{2}-125 \alpha^{3} \varphi^{3}+880 \alpha^{2} \beta^{2} \varphi-1720 \alpha^{2} \beta \varphi^{2}+800 \alpha^{2} \varphi^{3}\right. \\
& \left.-1260 \alpha^{2} \varphi^{2}-16 \alpha \beta^{2} \varphi-7488 \alpha \beta \varphi+5760 \alpha \varphi^{2}-576 \beta^{2}+10368 \varphi\right) /\left(72 X^{2}\right)
\end{aligned}
$$

Although this section pointed out the significance of the regulatory uncertainty on the incumbent's incentives to invest in NGA networks, a more elaborate analysis is needed in order to examine such relationship from a social perspective.

\subsection{Socially optimal investments}

The socially optimal investment level is derived by taking the first order condition of Eq. (22) with respect to $I$. Hence, the NGA investment level that maximizes social welfare is given by:

$$
I^{* *}=(1-c)(32 \beta-2 \alpha \varphi) /\left(36 \varphi+\alpha \varphi^{2}+4 \alpha \beta \varphi-32 \beta^{2}\right)
$$

Eq. (26) states that the NGA investment level that maximizes social welfare is positive as long as: (i) the investment cost parameter, $\varphi$, is not extremely high in relation to the impact of investments on demand (i.e. $\varphi<16 \beta / \alpha$ ); and (ii) social welfare is a concave function of $I$ (i.e. $Y \equiv 36 \varphi+\alpha \varphi^{2}+4 \alpha \beta \varphi-32 \beta^{2}>0$ ). A necessary and sufficient condition which ensures that $X$ and $Y$ are both positive is $\varphi>\varphi_{L}$, where $\varphi_{L}=\left(2 \sqrt{\alpha^{2} \beta^{2}+8 \alpha \beta^{2}+18 \alpha \beta+81}-2 \alpha \beta-18\right) / \alpha{ }^{6}$

Remark 1. Assuming that the investment cost parameter is higher than the critical value $\varphi_{L}$ ensures that the privately and the socially optimal investment levels are both positive as long as the investment cost parameter is not extremely high in relation to the impact of investments on demand.

Remark 1 implies that the higher the impact of the investments on demand, the higher the investment cost parameter should be in order to ensure that the incumbent will choose a positive NGA investment level that maximizes either its profits or social welfare. Therefore, given that the investment cost parameter is not extremely high in relation to the impact of investments on demand (i.e. $\varphi<16 \beta / \alpha$ ), the assumption $\varphi>\varphi_{L}$ ensures that $X, Y>0$.

Furthermore, taking the first derivative of Eq. (26) with respect to $\alpha$ yields:

$$
\partial I^{* *} / \partial \alpha=8 \varphi(c-1)\left(9 \varphi+8 \beta^{2}+4 \beta \varphi\right) /\left(Y^{2}\right)<0
$$

From Eq. (27) it can be deduced that there is a negative relationship between $\alpha$ and $I^{* *}$ since $c<1$. This implies that the socially optimal investment level increases with an increase in the probability of setting the access price equal to the marginal cost of providing the access. Therefore, a marginal increase in $\alpha$ positively affects the private investment incentives and negatively affects the socially optimal investments. This is an expected result since the incumbent's profits increase with an increase in the access price, whereas social welfare increases with a decrease in the access price.

However, for any given value of $\alpha$, the level of social welfare that corresponds to the socially optimal investments reflects the maximum social welfare outcome. This level is derived by substituting $I^{* *}$ into Eq. (22):

$$
S W^{* *}=\varphi(1-c)^{2}\left(288+8 \alpha \varphi+\alpha^{2} \varphi\right) /(18 Y)
$$

Since the aim of this paper is to study the impact of the regulatory uncertainty on an incumbent's incentives to undertake the socially optimal investments in NGA networks, the next section derives the value of $\alpha$ that results in the same privately and socially optimal investment level.

\footnotetext{
${ }^{6}$ The proof is given in Appendix A2.
} 


\section{Comparison of privately and socially optimal investments}

This section assesses the value of $\alpha$ that makes the incumbent undertake the socially optimal investments in NGA networks. The comparison of $I^{*}$ and $I^{* *}$ shows that:

$$
I^{* *}-I^{*}=3 Z \varphi(1-c) /(X Y)
$$

where

$$
Z=144 \beta+52 \alpha \beta \varphi-72 \alpha \varphi-5 \alpha^{2} \varphi^{2}-56 \alpha \beta^{2}
$$

Given that $X, Y>0$ and $c<1$, it can be concluded that when $Z=0$, the incumbent undertakes the socially optimal investments in NGA networks, whereas when $Z>0$ (respectively, $Z<0$ ) the incumbent underinvests (respectively, overinvests) compared to the socially optimal investment level.

In addition, the comparison of $S W^{*}$ and $S W^{* *}$ shows that:

$$
S W^{* *}-S W^{*}=[Z \varphi(1-c)]^{2} /\left(72 X^{2} Y\right)
$$

From Eq. (31) it can be inferred that the society is always better off when the incumbent undertakes the socially optimal investment level rather than the respective level that maximizes its profits, with the exception of $Z=0$. In the latter case, the incumbent undertakes the socially optimal investments, and hence, the derived social welfare level reaches the maximum welfare outcome. It can be thus deduced that the incumbent's choice to deviate from the socially optimal investment level results in welfare losses which reflects the standard trade-off between static and dynamic efficiency.

Solving Eq. (30) with respect to $\alpha$ derives the value of the probability of including a markup into the access price that tackles this efficiency trade-off. In other words, the value of $\alpha$ which leads to $I^{* *}=I^{*}$ and $S W^{* *}=S W^{*}$ (or equivalently to $Z=0$ ) is given by:

$$
\tilde{\alpha}=\left[\begin{array}{l}
26 \beta \varphi-36 \varphi-28 \beta^{2} \pm \\
2 \sqrt{196 \beta^{4}-364 \beta^{3} \varphi+169 \beta^{2} \varphi^{2}+504 \beta^{2} \varphi-288 \beta \varphi^{2}+324 \varphi^{2}}
\end{array}\right] /\left(5 \varphi^{2}\right)
$$

Let $\tilde{\alpha}$ denotes the positive value of $\alpha$ that induces the incumbent to undertake the socially optimal investments. ${ }^{7}$ Therefore:

$$
\tilde{\alpha}=\left[\begin{array}{l}
26 \beta \varphi-36 \varphi-28 \beta^{2}+ \\
2 \sqrt{196 \beta^{4}-364 \beta^{3} \varphi+169 \beta^{2} \varphi^{2}+504 \beta^{2} \varphi-288 \beta \varphi^{2}+324 \varphi^{2}}
\end{array}\right] /\left(5 \varphi^{2}\right)
$$

It can be thus deduced that, when $\alpha>\tilde{\alpha}$ (respectively, $\alpha<\tilde{\alpha}$ ), the value of $Z$ is negative (respectively, positive), and hence, the NGA investment level chosen by the incumbent is higher (respectively, lower) than the socially optimal one. This implies that any deviation from the socially optimal investments leads to welfare losses (i.e. $S W^{* *}>S W^{*}$ for $\alpha \neq \tilde{\alpha}$ ). The derived value of $\tilde{\alpha}$ is significantly affected by the impact of the investments on demand and the investment cost parameter. In particular, the value of $\tilde{\alpha}$ is positively affected by an increase in $\beta$ and negatively affected by an increase in $\varphi$ (ceteris paribus). ${ }^{8}$ This implies that, for a given investment cost parameter, higher consumers' valuation for the NGA services results in higher $\tilde{\alpha}$, which in turn leads to higher efficient investment levels. In other words, higher values of $\beta$, make the investments more socially desirable, and hence, the socially optimal investment level is achieved for a higher probability of compensating the incumbent for the investment risks. This result positively affects the incumbent's investment incentives, and hence, the achieved efficient investment level increases as well.

On the contrary, for a given positive impact of the investments on demand, a steeper slope of the marginal investment cost function leads to lower values of $\tilde{\alpha}$. This implies that as the NGA investments become marginally more expensive, the society is better off by a lower NGA deployment which is achieved by a higher probability of setting the access price at the marginal cost of providing the access. Therefore, the efficient NGA investment level is achieved for lower values of $\tilde{\alpha}$.

Fig. 1 graphically presents the results of the above analysis concerning the impact of $\beta$ and $\varphi$ on $\tilde{\alpha}$. In particular, it presents the results of the numerical simulations used in order to derive the values of $\tilde{\alpha}$ for different combinations of $\beta$ and $\varphi$ given that $\varphi>\varphi_{L}(\tilde{\alpha}){ }^{9}$

Fig. 1 graphically verifies the positive relationship between $\tilde{\alpha}$ and $\beta$, as well as, the negative relationship between $\tilde{\alpha}$ and $\varphi$. In addition, it is obvious that there are some combinations of $\beta$ and $\varphi$ which leads to $\tilde{\alpha}>1$. This implies that for every value of $\beta$ there is at least one value of $\varphi$ that makes $\tilde{\alpha}=1$. Solving $\tilde{\alpha}=1$ with respect to $\varphi$ yields:

$$
\tilde{\varphi}=\left(26 \beta-36 \pm 6 \sqrt{11 \beta^{2}-32 \beta+36}\right) / 5
$$

\footnotetext{
${ }^{7}$ This result is numerically proven in Appendix B. In particular, the positive (respectively, negative) value of $\tilde{\alpha}$ is decreasing (respectively, increasing) function of $\varphi$. However, there is always one positive and one negative value of $\tilde{\alpha}$ since they both tend to zero as $\varphi$ tends to infinity.

${ }^{8}$ The proof is given in Appendix A3.

${ }^{9}$ Fig. 1 is a graphical representation of Tables B1-B3 of Appendix B.
} 


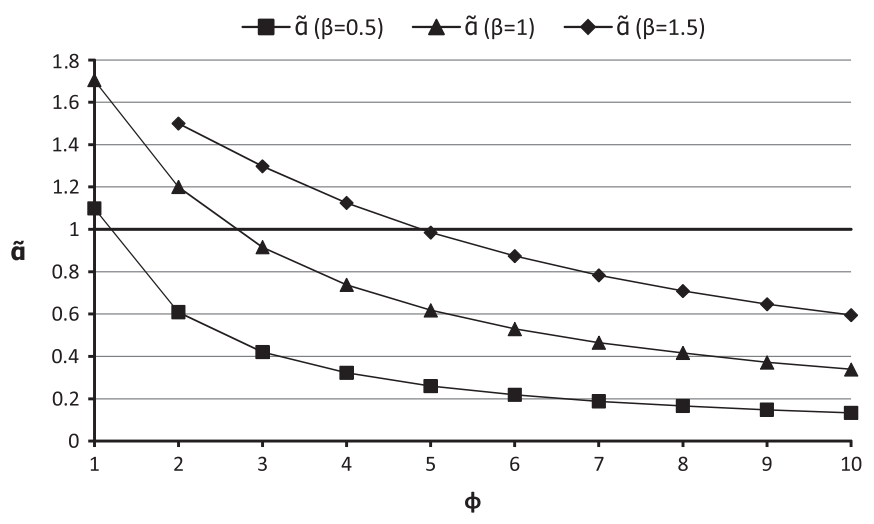

Fig. 1. The relationship between $\tilde{\alpha}$ and $\varphi$.

Let $\tilde{\varphi}$ denote the highest value of $\varphi$ that makes the privately and the socially optimal investment levels coincide when $\tilde{\alpha}=1 .{ }^{10}$ Therefore:

$$
\tilde{\varphi}=\left(26 \beta-36+6 \sqrt{11 \beta^{2}-32 \beta+36}\right) / 5
$$

From Eq. (35) it can be deduced that as long as $\varphi<\tilde{\varphi}$, the value of $\alpha$ that makes the incumbent undertake the socially optimal investments is higher than 1. In other words, when the slope of the marginal investment cost function is not particularly steep in relation to the impact of NGA investments on consumers' willingness to pay, then regulatory certainty about the incorporation of an access markup into the access price (i.e. $\alpha=1$ ) is not adequate to achieve the efficient investment outcome. This implies that the incumbent always underinvests compared to the socially optimal investment level since $\alpha \in[0,1]<\tilde{\alpha}$. In this case, a higher access markup which leads to $\tilde{\alpha} \leq 1$ seems to be socially desirable. This implies that the combination of a low investment cost parameter and a high impact of investments on demand makes the investments more attractive from a social perspective. Thus, the optimal regulatory policy is to provide the incumbent with significant investment incentives.

In addition, the critical value $\tilde{\varphi}$, which makes the incumbent undertake the socially optimal investments when $\tilde{\alpha}=1$, increases with an increasing rate as $\beta$ increases as well. ${ }^{11}$ This implies that as $\beta$ increases, the investment cost parameter should be significantly higher in order to ensure that $\tilde{\alpha}=1$. If the investment cost parameter is higher than the critical value of $\tilde{\varphi}$ given by Eq. (35), then the value of $\alpha$ that makes the incumbent undertake the socially optimal investments is lower than 1. In other words, when the investment cost parameter is relatively high compared to the impact of the investments on demand, the incumbent undertakes the socially optimal investments for a particular $\tilde{\alpha} \in(0,1)$. Therefore, the following proposition can be stated:

Proposition 1. As long as the investment cost parameter is high in relation to the impact of the investments on demand, there is a positive critical value of $\alpha$, denoted by $\tilde{\alpha} \in(0,1)$, that induces the incumbent to undertake the socially optimal investments. When $\alpha<\tilde{\alpha}$ (respectively, $\alpha>\tilde{\alpha}$ ), the incumbent underinvests (respectively, overinvests) compared to the socially optimal investments, and hence, there are welfare losses.

From Proposition 1, it can be deduced that the uncertainty about future regulatory intervention significantly affects the incumbent's expectations, and hence, its decision to undertake the socially efficient investments. Moreover, when the value of $\tilde{\alpha}$ is close to 0 , the regulator has significant incentives to set the access price at the marginal cost of providing the access. On the contrary, when the value of $\tilde{\alpha}$ is close to 1 , an investment-contingent access price which includes a lower markup than the average cost of the investments and leads to $\tilde{\alpha}=1$ seems to be socially desirable.

As it has been already stated above, the derived value of $\tilde{\alpha}$ is significantly affected by the particular value of the parameters $\beta$ and $\varphi$. Fig. 2 presents a representative example of the cases in which the difference between $\beta$ and $\varphi$ is relatively high (i.e. results in $\tilde{\alpha}<1$ ) since they illustrate the relationship between $I^{*}$ and $I^{* *}$, as well as, $S W^{*}$ and $S W^{* *}$ when $\beta=1$ and $\varphi=6$ (i.e. results in $\tilde{\alpha}=0.530$ ).

Fig. 2 numerically verifies the results of Eqs. (24) and (27) which state than an increase in $\alpha$ positively affects the privately investment incentives and negatively affects the socially optimal investments. In addition, it shows that the privately and the socially optimal NGA investment levels coincide for $\tilde{\alpha}=0.530<1$. This is due to the fact that the investment cost parameter is high in relation to the impact of the investments on demand since $\varphi=6>\tilde{\varphi}=2.648$. Therefore, when $\alpha=\tilde{\alpha}$, Eq. (30) yields $Z=0$, and hence, $I^{* *}=I^{*}$. On the contrary, when $\alpha<\tilde{\alpha}$ (respectively, $\alpha>\tilde{\alpha}$ ), the incumbent underinvests

\footnotetext{
10 Table B5 (see Appendix B) provides the values of $\tilde{\varphi}$ for different values of $\beta$. The discussion following Table B5 proves that: (i) the value of $\tilde{\varphi}$ given by Eq. (35) is the highest one; and (ii) the rejected value of $\tilde{\varphi}$ does not affect the final outcomes.

11 The proof is given in Appendix A4.
} 


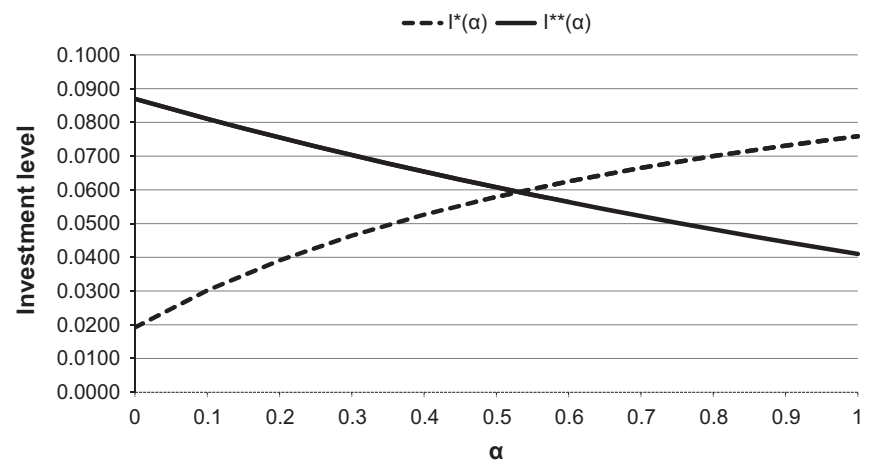

Fig. 2. The privately and socially optimal investment levels as a function of $\alpha(\beta=1, \varphi=6, c=0.5)$.

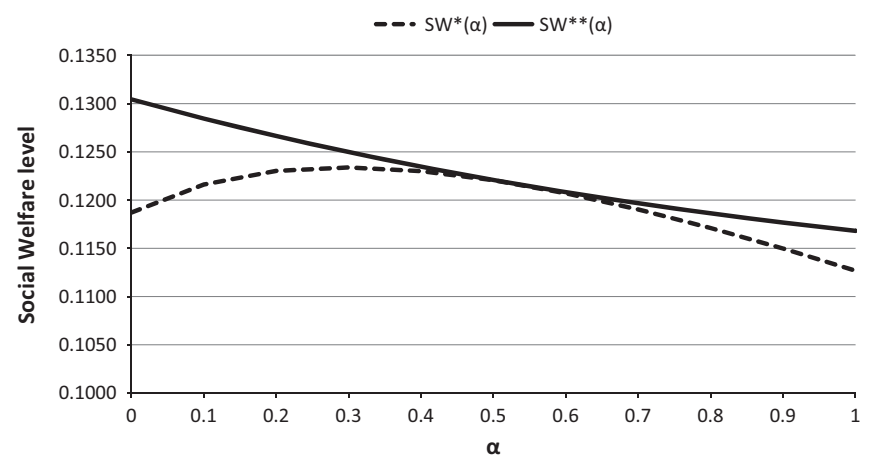

Fig. 3. The subsequent social welfare levels as a function of $\alpha(\beta=1, \varphi=6, c=0.5)$.

(respectively, overinvests) compared to the socially optimal investment level. The resulting social welfare levels are presented in Fig. 3.

Fig. 3 shows that there is a unique positive value of $\alpha$ which leads the social welfare levels derived by the privately and the socially optimal investment choices to coincide (i.e. $S W^{* * *}=S W^{*}$ ). In this numerical example, Eq. (31) holds for $\tilde{\alpha}=0.530$ which implies that the value of $\alpha$ that makes the incumbent undertake the socially optimal investments equals the respective value of $\alpha$ that makes the social welfare levels derived by the privately and the socially optimal investment choices coincide. Therefore, the incumbent not only undertakes the socially optimal investments, but also maximizes the potential social welfare outcome since $I^{* *}=I^{*}$ and $S W^{* *}=S W^{*}$. In all other cases $S W^{* *}>S W^{*}$, which means that the optimal social welfare outcome cannot be achieved with the incumbent's profit maximizing investment level.

\section{Conclusions}

The aim of this paper was to study the impact of regulatory uncertainty on an incumbent's incentives to undertake the socially optimal investments in NGA networks. For this reason, a regulatory non-commitment setting in which the incumbent invests prior to the regulation of the access was used. The related literature discusses the effectiveness of two different regulatory approaches on the regulator's goal to achieve the socially efficient investment level. The first approach supports that the regulator sets a particular investment-contingent access price, which compensates the incumbent for the investment risks, in order to provide significant investment incentives. On the contrary, the second approach argues that the regulator deviates from such ex ante known access price (once the investments are in place) by setting the access price at the marginal cost of providing the access (i.e. setting the social welfare maximizing access price).

The main contribution of this paper to the existing literature is that it modeled the more realistic case in which the regulator sets the access price at the marginal cost of providing the access with some probability and gives an access markup, which equals the average cost of the investments, with the complementary probability. Therefore, it is uncertain which of the two assumptions made in the related literature will prevail when the new access infrastructures are in place.

It is found that when the investment cost parameter $(\varphi)$ is not high (which implies that the slope of the marginal investment cost function is not particularly steep) in relation to the impact of investments on demand ( $\beta$ ), the incumbent underinvests compared to the socially optimal investment level. The reason is that the critical value of the probability of including an access markup into the access price $(\tilde{\alpha})$, which leads the incumbent to undertake the socially optimal investments, is decreasing in $\varphi$ and increasing in $\beta$. As a result, $\tilde{\alpha}>1$. This implies that the socially desirable outcome cannot 
be achieved even if the regulator commits to an access price scheme that includes an access markup equal to the average cost of the investments. In this case, a higher access markup which leads to $\tilde{\alpha} \leq 1$ seems to be socially desirable.

On the contrary, when the investment cost parameter is high and the impact of investments on demand is relatively low, the incumbent may overinvest or underinvest depending on the probability of incorporating the average cost of the investments into the access price. In this case, $\tilde{\alpha}<1$. In particular, when $\alpha<\tilde{\alpha}$ (respectively, $\alpha>\tilde{\alpha}$ ), the incumbent underinvests (respectively, overinvests) compared to the socially optimal investment, and hence, there are welfare losses. This implies that regulatory uncertainty significantly affects the incumbent's incentives to undertake the socially optimal investments in NGA networks.

However, it is acknowledged that significant future research is needed. For example, the derived results may change if we take into account the fact that the migration from copper access networks to NGA networks is a slow process (Bourreau, Cambini, \& Hoernig, 2012). This implies that during the migration phase both the legacy copper access networks and the NGA networks are in operation and are competing for customers. Therefore, the impact of the regulation of the legacy copper networks on the uncertainty about the regulation of the NGA networks should be modeled in order to assess the investment and competition outcomes. ${ }^{12}$

\section{Acknowledgments}

This research has been co-financed by the European Union (European Social Fund-ESF) and Greek national funds through the Operational Program "Education and Lifelong Learning” of the National Strategic Reference Framework (NSRF)_ Research Funding Program: Heracleitus II. Investing in knowledge society through the European Social Fund.

\section{Appendix A.}

A1. Proof that social welfare is maximized for $k<0$

Substituting Eqs. (9)-(11) into (1) and (2) gives the profit functions of the incumbent and the entrant, respectively, under Cournot competition:

$$
\begin{aligned}
& \pi_{1}=\left[2+2 \beta I(2+\beta I-7 c+5 w)+2 c^{2}-14 c+10 w(1+c-w)+9 \varphi I^{2}\right] / 18 \\
& \pi_{2}=(1+\beta I-2 w+c)^{2} / 9
\end{aligned}
$$

Consumer surplus is given by:

$$
C S=\left(q_{1}+q_{2}\right)^{2} / 2=(2+2 \beta I-c-w)^{2} / 18
$$

whereas social welfare $(S W)$ is the unweighted sum of industry profits and consumer surplus (i.e. $\left.S W=\pi_{1}+\pi_{2}+C S\right)$ :

$$
S W=\left(8+8 \beta^{2} I^{2}-14 \beta c I-2 \beta w I+16 \beta I+5 c^{2}+4 c w-14 c-w^{2}-2 w+9 \varphi I^{2}\right) / 18
$$

Now assume that the regulator sets an access price equal to the marginal cost of providing the access $(c)$ plus a fraction $k$ of the total investment costs, that is $w=c+k C(I)$, with $k<1$. Substituting this access price in Eq. (A4) and taking the first order condition with respect to $k$ gives:

$$
\begin{gathered}
\partial S W / \partial k=0 \Rightarrow-\varphi I^{2}\left(2+2 \beta I-2 c+k \varphi I^{2}\right) / 36=0 \Rightarrow \\
k^{*}=-2(1+\beta I-c) / \varphi I^{2}
\end{gathered}
$$

It is obvious that $k^{*}<0$ since $c<1$. This implies that the optimal access price is lower than the marginal cost of providing the access. Considering this optimal value of $k$, the access price is given by $w=2 c-\beta I-1$. Therefore, the retail price equals the marginal cost of providing the access $(p=c)$, the incumbent is not active in the market $\left(q_{1}=0\right)$ and the entrant produces the whole output $\left(q_{2}=1+\beta I-c\right)$. Since the incumbent's profits are negative, which are offset by the positive profits of the entrant, the optimal investment policy for the incumbent is to avoid any NGA deployment (i.e. $I=0$ given that $I \geq 0$ ).

However, it should be noted that since it is assumed that $w \geq c$, the regulator maximizes social welfare by setting $k^{*}=0$, or equivalently, $w=c$.

A2. Proof that $\varphi>\varphi_{L}$ is a necessary and sufficient condition to ensure that $X, Y>0$.

Recall that $X \equiv 18 \varphi+5 \alpha \varphi^{2}-4 \beta^{2}-10 \alpha \beta \varphi$ and $Y \equiv 36 \varphi+\alpha \varphi^{2}+4 \alpha \beta \varphi-32 \beta^{2}$. Therefore, $X=Y$ when:

$$
\varphi_{1}=\left(9+7 \alpha \beta-\sqrt{49 \alpha^{2} \beta^{2}-112 \alpha \beta^{2}+126 \alpha \beta+81}\right) /(4 \alpha)
$$

\footnotetext{
12 See Bourreau, Cambini, and Dogan (2012), Brito, Pereira, and Vareda (2010, 2012) and Inderst and Peitz (2012) for the impact of the regulation of the legacy network on the firms' investment incentives when the NGA market is left unregulated or when there is an interplay between the access prices of the two networks.
} 
or

$$
\varphi_{2}=\left(9+7 \alpha \beta+\sqrt{49 \alpha^{2} \beta^{2}-112 \alpha \beta^{2}+126 \alpha \beta+81}\right) /(4 \alpha)
$$

In particular, $Y>X$ when $\varphi_{1}<\varphi<\varphi_{2}$ and $X>Y$ when $\varphi<\varphi_{1}$ or $\varphi>\varphi_{2}$. In the former case $(Y>X), X$ and $Y$ are both positive if $X>0$. This implies that $\varphi$ should be higher than the positive root of $X=0$ with respect to $\varphi$ which is given by:

$$
\varphi_{3}=\left(5 \alpha \beta-9+\sqrt{25 \alpha^{2} \beta^{2}+20 \alpha \beta^{2}-90 \alpha \beta+81}\right) /(5 \alpha)
$$

However, note that the difference $\varphi_{1}-\varphi_{3}$ is always positive for $\beta>0$. This implies that $\varphi_{1}>\varphi_{3}$, and hence, $\varphi>\varphi_{3}$ since $\varphi_{1}<\varphi<\varphi_{2}$. As a result $X, Y>0$.

On the contrary, $X>Y$ when $\varphi<\varphi_{1}$ or $\varphi>\varphi_{2}$. In this case, $X$ and $Y$ are both positive if $Y>0$. This implies that $\varphi$ should be higher than the positive root of $Y=0$ with respect to $\varphi$ which is given by:

$$
\varphi_{4}=\left(2 \sqrt{\alpha^{2} \beta^{2}+8 \alpha \beta^{2}+18 \alpha \beta+81}-2 \alpha \beta-18\right) / \alpha
$$

It should be also noted that the difference $\varphi_{1}-\varphi_{4}$ is always positive for $\beta>0$. This implies that $\varphi_{4}<\varphi_{1}$. Therefore, the condition which ensures that $Y>0$ is $\varphi>\varphi_{4}$.

In conclusion, $\varphi>\varphi_{4} \equiv \varphi_{L}$, which implies that the investment cost parameter is higher than a critical value of $\varphi$ denoted by $\varphi_{L}$, is a necessary and sufficient condition which ensures that $X, Y>0$.

\section{A3. The impact of $\beta$ and $\varphi$ on $\tilde{\alpha}$.}

Taking the first order condition of $\tilde{\alpha}$ with respect to $\beta$ gives:

$$
\partial \tilde{\alpha} / \partial \beta=0 \Rightarrow \varphi_{\beta}=\left(13 \beta^{2}+36 \beta-\beta \sqrt{\left.169 \beta^{2}-576 \beta+1296\right)}\right) / 27
$$

Therefore, as long as $\varphi>\varphi_{\beta}$, there is a positive relationship between $\tilde{\alpha}$ and $\beta$. Substituting $\varphi_{\beta}$ into $\tilde{\alpha}$ and then the derived value of $\tilde{\alpha}$ into $\varphi_{L}$, gives the lower value of $\varphi$ which ensures that $X, Y>0$. It is proven that $\varphi_{\beta}=\varphi_{L}$, and hence $\partial \tilde{\alpha} / \partial \beta>0$ since $\varphi>\varphi_{L}$. The numerical simulations presented in Appendix B1 and graphically illustrated in Fig. 1 verify the positive relationship between $\tilde{\alpha}$ and $\beta$.

In addition, taking the first order derivative of $\tilde{\alpha}$ with respect to $\varphi$ gives:

$$
\frac{\partial \tilde{\alpha}}{\partial \varphi}=\left(\begin{array}{l}
18 \varphi \sqrt{\psi}-288 \beta \varphi^{2}+756 \beta^{2} \varphi-546 \beta^{3} \varphi-28 \beta^{2} \sqrt{\psi}+ \\
394 \beta^{4}+324 \varphi^{2}+169 \beta^{2} \varphi^{2}+13 \beta \varphi \sqrt{\psi}
\end{array}\right) /\left(5 \varphi^{3} \sqrt{\psi}\right)
$$

where $\psi=194 \beta^{4}-364 \beta^{3} \varphi+169 \beta^{2} \varphi^{2}+504 \beta^{2} \varphi-288 \beta \varphi^{2}+324 \varphi^{2}$. The numerical simulations presented in Appendix B1 and graphically illustrated in Fig. 1 show that there is a negative relationship between $\tilde{\alpha}$ and $\varphi$ (i.e. $\partial \tilde{\alpha} / \partial \varphi<0$ ).

\section{A4. The relationship between $\tilde{\varphi}$ and $\beta$}

Taking the first order derivative of $\tilde{\varphi}$ with respect to $\beta$ provides:

$$
\frac{\partial \tilde{\varphi}}{\partial \beta}=\left(\frac{33 \beta-48+13 \sqrt{11 \beta^{2}-32 \beta+36}}{5 \sqrt{11 \beta^{2}-32 \beta+36}}\right)
$$

It is easy to prove (i.e. using excel solver) that Eq. (A12) is minimized for $\beta=0$. Therefore, for $\beta>0$, there is a positive relationship between $\tilde{\varphi}$ and $\beta$. In addition, taking the second order derivative of $\tilde{\varphi}$ with respect to $\beta$ provides:

$$
\frac{\partial^{2} \tilde{\varphi}}{\partial \beta^{2}}=\frac{168}{\left(11 \beta^{2}-32 \beta+36\right)^{3 / 2}}
$$

It is obvious that the second order derivative of $\tilde{\varphi}$ with respect to $\beta$ is positive since $11 \beta^{2}-32 \beta+36>0$ due to Eq. (A12). Therefore, the critical value $\tilde{\varphi}$, which makes the incumbent undertake the socially optimal investments when $\tilde{\alpha}=1$, increases with an increasing rate as $\beta$ increases as well.

\section{Appendix B}

This section uses numerical simulations in order to assess the impact of regulatory uncertainty on the incumbent's incentives to undertake the socially optimal investment level. In particular, the positive value of $\alpha$, denoted by $\tilde{\alpha}$, that induces the incumbent to undertake the socially optimal investments (i.e. results in $Z=0$ ) is derived for many combinations of $\beta$ and $\varphi$ under the assumptions that: (i) $\varphi<16 \beta / \tilde{\alpha}$, which implies that the investment cost parameter is not extremely high in relation to the impact of investments on demand; and (ii) $\varphi>\varphi_{L}(\tilde{\alpha})$, which ensures that $I^{*}(\tilde{\alpha})>0$ and $I^{* * *}(\tilde{\alpha})>0$.

In addition, the analysis is limited to the cases in which $\beta<2$ in order to ensure that the entrant is always active in the retail market. In particular, substituting $I^{*}$ into Eq. (13) gives $q_{2}^{F C}=\varphi(1-c)(18-4 \beta-5 \alpha \beta) /(3 X)$. This implies that when the regulator compensates the incumbent for the investment risks, the entrant is active in the market when $(18-4 \beta-5 \alpha \beta)>0$. 
Obviously, $q_{2}^{F C}>0$ if $\alpha<(18-4 \beta) /(5 \beta)$. However, $q_{2}^{F C}$ decreases with an increase in $\alpha$. Therefore, if $q_{2}^{F C}>0$ when $\alpha=1$, then $q_{2}^{F C}>0$ for $\alpha \in[0,1]$. The condition which ensures that $q_{2}^{F C}>0$ when $\alpha=1$ is $\beta<2$. Therefore, although a necessary and sufficient condition to ensure that $q_{2}^{F C}>0$ is $(18-4 \beta-5 \alpha \beta)>0$, the condition $\beta<2$ can be used for simplicity without affecting the final results.

Tables B1-B3 provide the positive and the negative values of $\alpha$, which are denoted respectively by $\tilde{\alpha}$ and $\tilde{\alpha}_{2}$, and lead the incumbent to undertake the socially optimal investments. The derive results corresponds to the cases in which the investment cost parameter takes positive integral values between 1 and 10, whereas the impact of investment on demand is $\beta=0.5, \beta=1$ or $\beta=1.5$. It is found that regardless of the particular value of $\beta, \tilde{\alpha}$ is always positive and $\tilde{\alpha}_{2}$ is always negative. In addition, $\tilde{\alpha}$ is positively correlated with $\beta$ and negatively correlated with $\varphi$. These results numerically verify the respective results of Appendix A3. Moreover, an increase in $\varphi$ leads $\tilde{\alpha}$ to decrease and $\tilde{\alpha}_{2}$ to increase. Simulations show that when $\varphi$ tends to infinity, then $\tilde{\alpha}=\tilde{\alpha}_{2}=0$. This implies that there is always one positive and one negative value of $\tilde{\alpha}$. Thus, the whole analysis is based on the positive value of $\alpha$, denoted by $\tilde{\alpha}$, which induces the incumbent to undertake the socially optimal investments. Tables B1-B3 also show the value of $\varphi$, denoted by $\varphi_{L}(\tilde{\alpha})$, which ensure that when $\alpha=\tilde{\alpha}$, the privately and the socially optimal investment levels are both positive. The derived levels of investments and social welfare are also provided for $\varphi>\varphi_{L}(\tilde{\alpha})$.

It should be noted that for $\tilde{\alpha} \in[0,1]$ the optimal investment level is lower than 1 . This result leads to access prices which are higher than the total investment costs, and hence, the condition that $k<1$ is violated. However, this result is due to the

Table B1

\begin{tabular}{|c|c|c|c|c|c|}
\hline$\varphi$ & $\tilde{\alpha}$ & $\tilde{\alpha}_{2}<0$ & $\varphi_{L}(\tilde{\alpha})$ & $I^{*}(\tilde{\alpha})=I^{* *}(\tilde{\alpha})$ & $S W^{*}(\tilde{\alpha})=S W^{* *}(\tilde{\alpha})$ \\
\hline 1 & 1.099 & -13.099 & 0.208 & 0.220 & 0.132 \\
\hline 2 & 0.609 & -5.909 & 0.214 & 0.098 & 0.120 \\
\hline 3 & 0.421 & -3.799 & 0.217 & 0.063 & 0.117 \\
\hline 4 & 0.322 & -2.797 & 0.218 & 0.047 & 0.115 \\
\hline 5 & 0.260 & -2.212 & 0.219 & 0.037 & 0.115 \\
\hline 6 & 0.219 & -1.830 & 0.219 & 0.031 & 0.114 \\
\hline 7 & 0.188 & -1.560 & 0.220 & 0.026 & 0.114 \\
\hline 8 & 0.166 & -1.359 & 0.220 & 0.023 & 0.113 \\
\hline 9 & 0.148 & -1.209 & 0.220 & 0.020 & 0.113 \\
\hline 10 & 0.133 & -1.081 & 0.220 & 0.028 & 0.113 \\
\hline
\end{tabular}

Table B2

$(\beta=1, c=0.5)$.

\begin{tabular}{|c|c|c|c|c|c|}
\hline$\varphi$ & $\tilde{\alpha}$ & $\tilde{\alpha}_{2}<0$ & $\varphi_{L}$ & $I^{*}(\tilde{\alpha})=I^{* * *}(\tilde{\alpha})$ & $S W^{*}(\tilde{\alpha})=S W^{* * *}(\tilde{\alpha})$ \\
\hline 1 & 1.704 & -16.904 & 0.726 & 1.142 & 0.338 \\
\hline 2 & 1.200 & -6.000 & 0.767 & 0.250 & 0.158 \\
\hline 3 & 0.916 & -3.494 & 0.792 & 0.133 & 0.137 \\
\hline 4 & 0.738 & -2.428 & 0.809 & 0.096 & 0.129 \\
\hline 5 & 0.618 & -1.860 & 0.821 & 0.072 & 0.124 \\
\hline 6 & 0.530 & -1.508 & 0.830 & 0.059 & 0.122 \\
\hline 7 & 0.465 & -1.265 & 0.837 & 0.050 & 0.120 \\
\hline 8 & 0.417 & -1.088 & 0.842 & 0.043 & 0.119 \\
\hline 9 & 0.372 & -0.955 & 0.846 & 0.038 & 0.118 \\
\hline 10 & 0.339 & -0.851 & 0.850 & 0.034 & 0.117 \\
\hline
\end{tabular}

Table B3

$(\beta=1.5, c=0.5)$.

\begin{tabular}{|c|c|c|c|c|c|}
\hline$\varphi$ & $\tilde{\alpha}$ & $\tilde{\alpha}_{2}<0$ & $\varphi_{L}$ & $I^{*}(\tilde{\alpha})=I^{* *}(\tilde{\alpha})$ & $S W^{*}(\tilde{\alpha})=S W^{* *}(\tilde{\alpha})$ \\
\hline 1 & 1.682 & -25.622 & 1.482 & - & - \\
\hline 2 & 1.500 & -7.200 & 1.523 & 0.875 & 0.366 \\
\hline 3 & 1.298 & -3.698 & 1.571 & 0.283 & 0.190 \\
\hline 4 & 1.125 & -2.400 & 1.616 & 0.167 & 0.156 \\
\hline 5 & 0.985 & -1.753 & 1.654 & 0.118 & 0.142 \\
\hline 6 & 0.874 & -1.374 & 1.686 & 0.091 & 0.135 \\
\hline 7 & 0.783 & -1.126 & 1.713 & 0.074 & 0.130 \\
\hline 8 & 0.709 & -0.952 & 1.736 & 0.062 & 0.127 \\
\hline 9 & 0.647 & -0.825 & 1.755 & 0.054 & 0.125 \\
\hline 10 & 0.595 & -0.727 & 1.772 & 0.047 & 0.123 \\
\hline
\end{tabular}


Table B4

$(\beta=2, c=0.5)$.

\begin{tabular}{llllr}
\hline$\varphi$ & $\tilde{\alpha}$ & $\tilde{\alpha}_{2}<0$ & $\varphi_{L}$ & $I^{*}(\tilde{\alpha})=I^{* * *}(\tilde{\alpha})$ \\
\hline $\mathbf{9}$ & 0.926 & -0.768 & 2.783 & 0.070 \\
$\mathbf{1 0}$ & 0.861 & -0.669 & 2.824 & 0.134 \\
\hline
\end{tabular}

Table B5

$(c=0.5)$.

\begin{tabular}{|c|c|c|c|c|c|c|}
\hline$\beta$ & 0.5 & 1 & 1.5 & 2 & 2.5 & 3 \\
\hline$\tilde{\varphi}$ & 1.124 & 2.648 & 4.885 & 8 & 11.770 & 15.894 \\
\hline$\tilde{\varphi}_{2}$ & -10.324 & -6.648 & -3.685 & -1.6 & -0.170 & 0.906 \\
\hline
\end{tabular}

normalized demand function used in this paper. It is easy to proof that an inverse demand function given (for example) by $p=100+\beta I-\left(q_{1}+q_{2}\right)$, which implies that this demand curve intersects the price axis at a significantly higher point than $p=1+\beta I-\left(q_{1}+q_{2}\right)$, results in $k<1$ without affecting the derived outcomes concerning the particular values of $\tilde{\alpha}$.

Table B4 presents the corresponding results when $\beta=2$. In this case the value of $\alpha$ should be lower that 1 in order to ensure that $q_{2}^{F C}>0$. It is found that $\tilde{\alpha}<1$ for $\varphi>8$. In addition, when $\beta=2.5, \tilde{\alpha} \leq 1$ for $\varphi \geq 21.25$. This implies that as $\beta$ increases, the level of the investment cost parameter which ensures that $q_{2}^{F C}>0$ increases with an increasing rate.

Lastly, Table B5 shows the values of $\tilde{\varphi}$ that makes the privately and the socially optimal investment levels coincide when $\tilde{\alpha}=1$.

It is obvious that as long as $\beta \leq 2.5$, the highest value of $\varphi$ that makes the privately and the socially optimal investment levels coincide when $\tilde{\alpha}=1$ is denoted by $\tilde{\varphi}$ since $\tilde{\varphi}>0$ and $\tilde{\varphi}_{2}<0$. Therefore, if $\varphi<\tilde{\varphi}$ (respectively, $\varphi>\tilde{\varphi}$ ), the value of $\alpha$ that makes the incumbent undertake the socially optimal investments is higher (respectively, lower) than 1 . However, note that when $\beta=2$, the value of $\tilde{\varphi}$ is equal to the respective value of $\varphi$ that makes $q_{2}^{F C}=0$. Therefore, $\varphi>\tilde{\varphi}$, and hence, $\tilde{\alpha}<1$. In addition, when $\beta \geq 3, \tilde{\varphi}$ and $\tilde{\varphi}_{2}$ are both positive. However, note that in such cases the value of $\varphi$ should be extremely high in order to ensure that $q_{2}^{F C}>0$. For example, when $\beta=3$, the investment cost parameter which ensure that $q_{2}^{F C}>0$ is $\varphi>48$. Therefore, $\varphi$ should be higher than the highest value of $\varphi$ that makes the privately and the socially optimal investment levels coincide when $\tilde{\alpha}=1$. As a result, $\varphi>\tilde{\varphi}$, which implies that $\tilde{\alpha}<1$. From the above analysis, it is proven that $\tilde{\varphi}_{2}$ does not affect the final outcomes, and hence, the whole analysis should focus on $\tilde{\varphi}$.

Furthermore, Table B5 numerically verifies the results of Appendix A4 concerning the relationship between $\beta$ and $\tilde{\varphi}$. In particular, the critical value $\tilde{\varphi}$ increases with an increasing rate as $\beta$ increases as well. In addition, Table B5 is in line with Fig. 1 since it reveals the values of $\varphi$ that make $\tilde{\alpha}=1$ for different values of $\beta$.

\section{References}

Bender, C. (2011). How to provide access to next generation networks? The effect of risk allocation on investment and cooperation incentives. Available from: 〈http://ideas.repec.org/p/mar/magkse/201149.html〉.

Bouckaert, J., van Dijk, T., \& Verboven, F. (2010). Access regulation, competition, and broadband penetration: An international study. Telecommunications Policy, 34(11), 661-671.

Bourreau, M., Cambini, C., \& Dogan, P. (2012). Access pricing, competition, and incentives to migrate from ‘old' to 'new' technology. International Journal of Industrial Organization, 30(6), 713-723.

Bourreau, M., Cambini, C., \& Hoernig, S. (2012). Ex ante regulation and co-investment in the transition to next generation access. Telecommunications Policy, 36(5), 399-406.

Brito, D., Pereira, P., \& Vareda, J. (2010). Can two-part tariffs promote efficient investment on next generation networks?. International Journal of Industrial Organization, 28(3), 323-333.

Brito, D., Pereira, P., \& Vareda, J. (2012). Incentives to invest and to give access to non-regulated new technologies. Information Economics and Policy, 24(3-4), $197-211$.

Cambini, C., \& Jiang, Y. (2009). Broadband investment and regulation: A literature review. Telecommunications Policy, 33(10-11), 559-574.

Cambini, C., \& Silvestri, V. (2012). Technology investment and alternative regulatory regimes with demand uncertainty. Information Economics and Policy, 24 (3-4), 212-230.

Cave, M., \& Prosperetti, L. (2001). European telecommunications infrastructures. Oxford Review of Economic Policy, 17(3), 416-431.

EU Commission (2010). Commission recommendation on regulated access to next generation access networks (NGA). Available from: 〈http://eur-lex. europa.eu/LexUriServ/LexUriServ.do?uri=OJ:L:2010:251:0035:0048:EN:PDF>.

Foros, O. (2004). Strategic investments with spillovers, vertical integration and foreclosure in the broadband access market. International Journal of Industrial Organization, 22(1), 1-24.

Henriques, D. (2011). Can access price indexation promote efficient investment in next generation networks? Available from: 〈http://ssrn.com/ abstract $=1955209\rangle$.

Inderst, R., \& Peitz, M. (2012). Market asymmetries and investments in next generation access networks. Review of Network Economics, 11(1).

Jorde, T., Sidak, G., \& Teece, D. (2000). Innovation, Investment and Unbundling. Yale Journal on Regulation, 17(1), 1-37.

Klumpp, T., \& Su, X. (2010). Open access and dynamic efficiency. American Economic Journal: Microeconomics, 2, 64-96.

Kotakorpi, K. (2006). Access price regulation, investment and entry in telecommunications. International Journal of Industrial Organization, 24(5), 1013-1020.

Mizuno, K., \& Yoshino, I. (2012). Distorted access regulation with strategic investments: Regulatory non-commitment and spillovers revisited. Information Economics and Policy, 24(2), 120-131. 
Nitsche, R., \& Wiethaus, L. (2011). Access regulation and investment in next generation networks-A ranking of regulatory regimes. International Journal of Industrial Organization, 29(2), 263-272.

OPTA (2010). Regulation, risk and investment incentives, Regulatory policy note 06. Available from: 〈http://www.opta.nl/en/news/all-publications/ publication/?id=3201 $>$.

Sarmento, P., \& Brandao, A. (2007). Access pricing: A comparison between full deregulation and two alternative instruments of access price regulation, costbased and retail-minus. Telecommunications Policy, 31(5), 236-250.

Sauer, D. (2011). Access rules and investment incentives. Available from: 〈http://innovation-regulation2.telecom-paristech.fr/wp-content/uploads/2011/11/ Sauer.pdf $\rangle$.

Valletti, T. M. (2003). The theory of access pricing and its linkage with investment incentives. Telecommunications Policy, 27(10-11), 659-675.

Vareda, J. (2010). Access regulation and the incumbent investment in quality-upgrades and in cost-reduction. Telecommunications Policy, 34(11), 697-710.

WIK (2009). The economics of next generation access. A study for the European competitive telecommunications association ECTA. Available from: 〈http:// www.wik.org/uploads/media/Ecta_Study_Addendum_2009.pdf). 